\title{
Neoadjuvant vs definitive concurrent chemoradiotherapy in locally advanced esophageal squamous cell carcinoma patients
}

\author{
Chih-Yi Chen ${ }^{1+}$, Chia-Chin $\mathrm{Li}^{2+}$ and Chun-Ru Chien ${ }^{2,3,4^{*}}$ (1)
}

\begin{abstract}
Background: The optimal treatment for locally advanced esophageal squamous cell carcinoma remains unclear. We compared the clinical outcomes of neoadjuvant concurrent chemoradiotherapy (CCRT) followed by esophagectomy [the surgery group] and CCRT without surgery [the CCRT group] in patients with squamous cell carcinoma from an Asian population.

Methods: Eligible patients diagnosed from 2008 to 2015 were identified through the Taiwan Cancer Registry. To balance observable potential confounders, we constructed a 1:1 propensity score-matched cohort [surgery vs CCRT]. We compared the hazard ratios between the surgery and CCRT groups for death using a robust variance estimator. We also evaluated the outcomes of patients for freedom from local regional recurrence (FFLRR) and esophageal cancer-specific survival (ECSS). Extensive supplementary analyses were performed to examine the robustness of our findings.
\end{abstract}

Results: Our study population included 298 patients balanced with respect to the observed covariables. The hazard ratio of death was 0.56 [95\% confidence interval 0.42 0.75] when surgery was compared to CCRT. The results remained significant in the FFLRR and ECSS outcomes. In the supplementary analyses, our results also remained significant when additional covariables were taken into consideration or when the definition of the index date was changed.

Conclusions: When compared to definitive CCRT, neoadjuvant CCRT followed by esophagectomy was associated with improved overall survival for locally advanced esophageal squamous cell carcinoma. However, given the nonrandomized nature of the study and the sensitivity to potentially unmeasured confounders, our results should be interpreted cautiously.

Keywords: Esophageal squamous cell carcinoma, Concurrent chemoradiotherapy, Esophagectomy

\section{Background}

Esophageal cancer is a common cause for cancer mortality around the world [1], and except in North America and Europe, squamous cell carcinoma (SqCC) is the major histological subtype [1].

\footnotetext{
* Correspondence: d16181@gmail.com

${ }^{+}$Chih-Yi Chen and Chia-Chin Li contributed equally to this work.

${ }^{2}$ Department of Radiation Oncology, China Medical University Hospital, Taichung, Taiwan

${ }^{3}$ Department of Radiation Oncology, China Medical University Hsinchu Hospital, Hsinchu, Taiwan

Full list of author information is available at the end of the article
}

The optimal treatment for locally advanced esophageal $\mathrm{SqCC}$ has remained elusive. According to the current National Comprehensive Cancer Network guidelines, esophagectomy, neoadjuvant concurrent chemoradiotherapy (CCRT) followed by esophagectomy, or definite CCRT were all possible treatment options for cT1b-4aN0-+M0 patients [2]. A seminal paper published in the New England Journal of Medicine in 2014 states that "locally advanced tumors, defined as category T3N1, are best treated with esophagectomy" [1]. However, the role of esophagectomy was questioned in a review paper published in 2017 [3]. Meta-analyses and a recent large-scale randomized

(c) The Author(s). 2018 Open Access This article is distributed under the terms of the Creative Commons Attribution 4.0 International License (http://creativecommons.org/licenses/by/4.0/), which permits unrestricted use, distribution, and 
controlled trial (RCT) reported favorable outcomes when neoadjuvant CCRT followed by esophagectomy was compared to esophagectomy alone $[4,5]$. In addition, RCTs for esophageal SqCC patients from Germany and France had reported similar overall survival (OS), although better local control was obtained with neoadjuvant CCRT followed by esophagectomy compared to CCRT without surgery (also mentioned in the 2017 review paper above) $[3,6,7]$. Another RCT also reported similar OS between CCRT without surgery and upfront esophagectomy without neoadjuvant CCRT [8].

Therefore, the aim of our study was to compare neoadjuvant CCRT followed by esophagectomy to definitive CCRT for locally advanced esophageal SqCC patients in a real-world Asian population.

\section{Methods}

\section{Data source}

In our study, the primary data comes from the Taiwan Cancer Registry (TCR) and death registration. The TCR is a high-quality database [9] that provides complete information such as individual demographics, stage of disease, tumor histology, and treatment details. Some prognostic factors, e.g., the use of positron emission tomography (PET), were also available since 2011.

\section{Study population and study design}

Our study flow chart, designed to conform to the STROBE guidelines [10], is depicted in Fig. 1. The main study population consisted of locally advanced esophageal SqCC patients diagnosed from 2008 to 2015 who received neoadjuvant CCRT [radiotherapy dose 40-50.4 Gy] before esophagectomy, or CCRT [radiotherapy dose $\geq 50.4 \mathrm{~Gy}$ ] without surgery. The explanatory variable of interest in this study was the surgery group (neoadjuvant CCRT followed by esophagectomy) vs the CCRT group (CCRT without surgery). We collected covariables based on our experiences in clinical care and related TCR studies [11-13] for adjustment of potential nonrandomized treatment selection (as mentioned in the next section). We defined the date of diagnosis from the cancer registry as the index date and obtained the survival statuses of patients from the death registry [follow-up until Dec 31, 2016]. We then estimated propensity scores (PSs) using the covariables to construct a PS-matched sample. We used this PS-based method rather than a Cox regression model, as suggested in previous literature $[14,15]$.

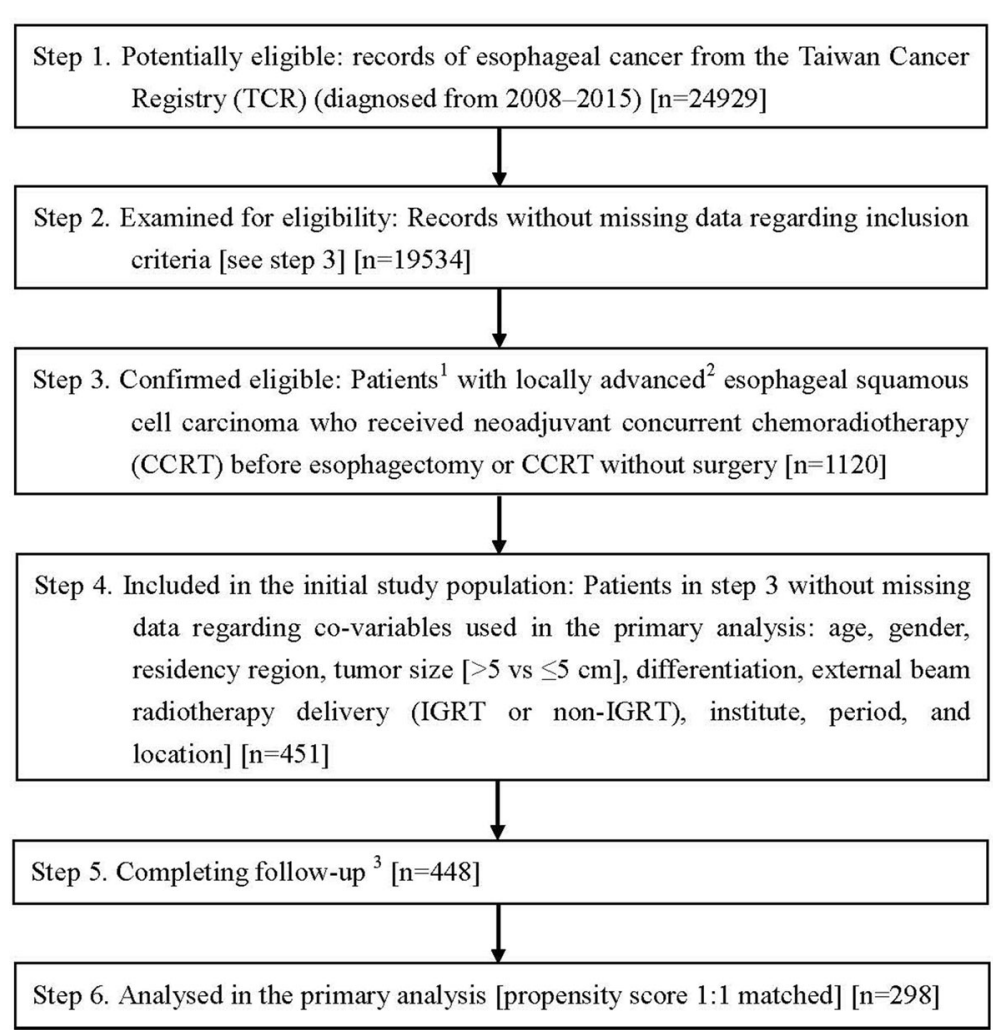

Fig. 1 STROBE study flow chart and the numbers of individuals at each stage of the study. 1: We only included those treated (classes 1-2) by any single institution to ensure data consistency. 2: Cancer staging clinical stage T3N1M0. 3: Without missing information in the TCR and the death registry 


\section{Other explanatory covariables}

Patient demographics [age, gender, residency region], disease characteristics [tumor size, differentiation, tumor location], radiotherapy (RT) delivery, institution, and period were included in our primary analysis. We also considered a prognostic factor ["use of PET", available in the TCR since 2011] in the supplementary analyses. The definitions of covariables were as follows. Patient residency region was classified as northern Taiwan or elsewhere. Tumor size was classified by a diameter $\leq 5$ or $>5 \mathrm{~cm}$. Tumor differentiation was classified as well/moderately differentiated or poorly/undifferentiated. External beam radiotherapy delivery was classified as image-guided radiotherapy (IGRT) or non-IGRT. The hospital was classified as a high- or low-volume institute via a threshold [20 esophagectomies per year] [16]. The time period was classified as 2008-2009 or 2010-2015. Tumor location was classified as cervical or not.

\section{Statistical analysis}

We performed the statistical analysis using the software SAS 9.4 (SAS Institute, Cary, NC, USA) and STATA 12 (StataCorp LP, College Station, TX, USA). In the primary analysis, we used a logistic regression model based on covariables to evaluate the probability of receiving surgery (vs CCRT) and then used the logit of the probability as the PS in a PS-matched method. Tabulation and standardized difference $[17,18]$ were used to assess the balance of covariates between PS-matched groups. We used a robust variance estimator to compare the hazard ratios of events between surgery- and CCRT-matched groups during the entire follow-up period [14]. As suggested in the recent literature [19], we evaluated the robustness of our findings to potential unmeasured confounding factor(s) via the E-factor. We also evaluated the outcomes of patients for freedom from local regional recurrence (FFLRR) and esophageal cancer-specific survival (ECSS) according to the TCR and the death registry.

\section{Supplementary analysis}

In a subgroup of patients [diagnosed from 2011 to 2015] for whom additional information, i.e., use of PET, was available, we performed the first supplementary analysis (SA-1). In the second supplementary analysis (SA-2), we repeated what we did in SA-1 but limited the surgery group to those who received minimally invasive esophagectomy (MIE) because of its potential superiority [20,21]. In the third supplementary analysis (SA-3), we reanalyzed the OS in the primary analysis when the index date was changed to the start of radiotherapy.

\section{Results}

Identification of the study population used in the primary analysis

As shown in Fig. 1, the identified initial study population consisted of 451 esophageal SqCC cancer patients divided into surgery or CCRT groups. After excluding the missing data in follow-up and applying a PS matching method, 298 patients were used as the final study population in the primary analysis. The patient characteristics are described in Table 1. All covariables after matching were well balanced with small standardized differences $(<0.25)$ [17].

\section{Primary analysis}

After a median follow-up of 20 months [range 3-98], death was observed for 79 patients in the surgery group and for 108 in the CCRT group. The hazard ratio (HR) of death when surgery was compared to CCRT was 0.56 [95\% confidence interval (95CI) $0.42-0.75, p$ value $<0.001$ ] The observed HR 0.56 for OS could be explained by an unmeasured confounder associated with the selection of treatment (IMRT or 3DCRT) and live/death by a risk ratio of 2.35-fold each; however, weaker confounding factors could not do so. Furthermore, the confidence interval could be moved to include the null hypothesis by an unmeasured confounder by a risk ratio of 1.74 , above and beyond the measured confounders; however, weaker confounding could not. The 5 -year OS rate for surgery was 38\% (vs 20\% for CCRT). Figure 2 shows the Kaplan-Meier survival curve for OS. Surgery was also associated with better FFLRR [HR 0.17 , 95CI $0.11-0.28, p$ value <.001] and ECSS [HR 0.56, 95CI 0.41-0.77, $p$ value $<.001]$.

\section{Supplementary analyses}

In the SA-1, which incorporated the use of PET into the PS model, all of the covariables were well balanced and the standardized differences were small $(<0.25)$ (Table 2). From this analysis, we found that surgery was still associated with improved OS compared to CCRT [HR 0.51, 95CI $0.36-0.72, p$ value $<0.001]$. Figure 3 shows the Kaplan-Meier survival curve for OS.

In the SA-2, in which neoadjuvant CCRT followed by MIE was compared to CCRT without surgery, all of the covariables were still well balanced with small standardized differences $(<0.25)$ (Table 3$)$. The HR of death when surgery [MIE] was compared to CCRT was 0.44 [95CI $0.28-0.70, p$ value $<0.001]$. Figure 4 shows the Kaplan-Meier survival curve for OS.

In the SA-3 in which we used the RT-start date as the index date, the HR for death when surgery was compared with CCRT was similar [0.55, 95CI 0.41-0.74, $p$ value $<0.001]$. The Kaplan-Meier survival curves for OS are shown in Fig. 5.

\section{Discussion}

In this population-based study PS-matched analysis from Asia (Taiwan), we found that for locally advanced esophageal SqCC, neoadjuvant CCRT followed by esophagectomy was associated with improved OS when compared to CCRT without surgery. 
Table 1 Characteristics of matched study population in the primary analysis

\begin{tabular}{|c|c|c|c|c|c|c|}
\hline & & Surgery & & CCRT & & Standardized \\
\hline & & Number or mean $(s d)^{a}$ & $(\%)^{a}$ & Number or mean $(\mathrm{sd})^{\mathrm{a}}$ & $(\%)^{\mathrm{a}}$ & \\
\hline Age & & $56.93(8.65)$ & & $57.26(10.48)$ & & 0.04 \\
\hline Gender & Female & 8 & (5) & 7 & (5) & 0.03 \\
\hline & Male & 141 & (95) & 142 & $(95)$ & \\
\hline Residency & Non-north & 101 & $(68)$ & 100 & $(67)$ & 0.01 \\
\hline & North & 48 & $(32)$ & 49 & (33) & \\
\hline Tumor size & $\leq 5 \mathrm{~cm}$ & 59 & $(40)$ & 60 & $(40)$ & 0.01 \\
\hline & $>5 \mathrm{~cm}$ & 90 & $(60)$ & 89 & $(60)$ & \\
\hline Differentiation & Poorly/undifferentiated & 46 & (31) & 53 & (36) & 0.10 \\
\hline & Well/moderately & 103 & $(69)$ & 96 & (64) & \\
\hline RT delivery & Non-IGRT & 131 & (88) & 128 & $(86)$ & 0.06 \\
\hline & IGRT & 18 & $(12)$ & 21 & $(14)$ & \\
\hline Institution & Low volume & 50 & (34) & 54 & (36) & 0.06 \\
\hline & High volume & 99 & $(66)$ & 95 & (64) & \\
\hline Period & 2008-2009 & 18 & $(12)$ & 19 & (13) & 0.02 \\
\hline & 2010-2015 & 131 & (88) & 130 & $(87)$ & \\
\hline Location & Cervical & 1 & (1) & 1 & (1) & 0.00 \\
\hline & Noncervical & 148 & (99) & 148 & (99) & \\
\hline
\end{tabular}

sd standard deviation, $R T$ radiotherapy, IGRT image-guided RT, CCRT concurrent chemoradiotherapy

${ }^{\mathrm{a}}$ Rounded

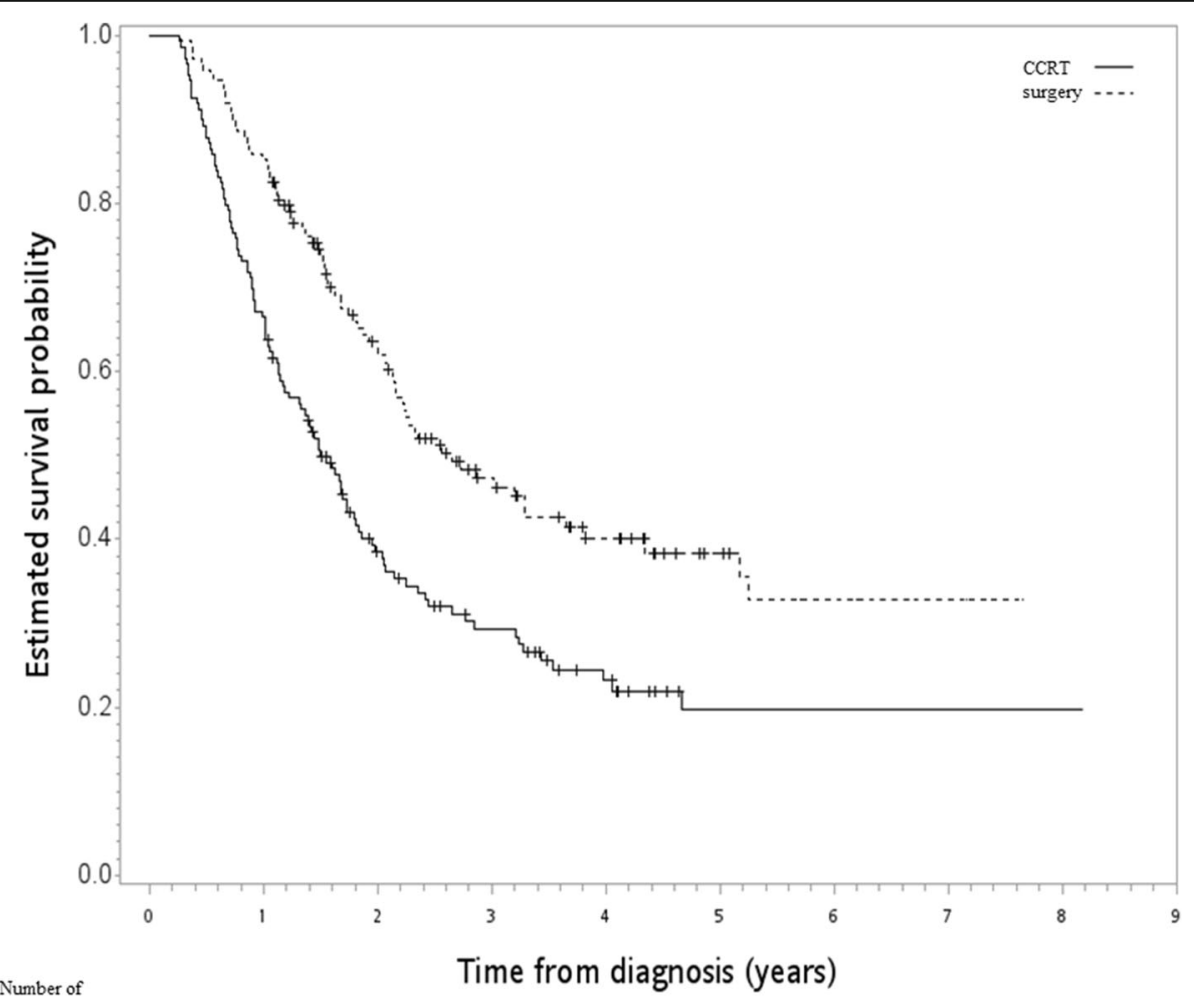

Number of

patients at risk

$\begin{array}{lrrrrrrrrr}\text { CCRT } & 149 & 99 & 48 & 32 & 19 & 6 & 5 & 3 & 2 \\ \text { surgery } & 149 & 127 & 77 & 44 & 29 & 16 & 10 & 5 & 0\end{array}$

Fig. 2 Kaplan-Meier survival curve (in years) for the final study population [primary analysis] 
Table 2 Characteristics of the matched study population in the SA-1

\begin{tabular}{|c|c|c|c|c|c|c|}
\hline & & \multicolumn{2}{|l|}{ Surgery } & \multicolumn{2}{|l|}{ CCRT } & \multirow{2}{*}{$\begin{array}{l}\text { Standardized } \\
\text { difference }^{a}\end{array}$} \\
\hline & & Number or mean $(s d)^{a}$ & $(\%)^{a}$ & Number or mean $(s d)^{a}$ & $(\%)^{\mathrm{a}}$ & \\
\hline Age & & $57.00(7.63)$ & & $56.61(9.62)$ & & 0.05 \\
\hline \multirow[t]{2}{*}{ Gender } & Female & 6 & $(5)$ & 3 & (3) & 0.14 \\
\hline & Male & 104 & (95) & 107 & $(97)$ & \\
\hline \multirow[t]{2}{*}{ Residency } & Non-north & 76 & (69) & 80 & (73) & 0.08 \\
\hline & North & 34 & (31) & 30 & $(27)$ & \\
\hline \multirow[t]{2}{*}{ Tumor size } & $\leq 5 \mathrm{~cm}$ & 43 & (39) & 42 & (38) & 0.02 \\
\hline & $>5 \mathrm{~cm}$ & 67 & $(61)$ & 68 & $(62)$ & \\
\hline \multirow[t]{2}{*}{ Differentiation } & Poorly/undifferentiated & 41 & $(37)$ & 42 & (38) & 0.02 \\
\hline & Well/moderately & 69 & (63) & 68 & $(62)$ & \\
\hline \multirow[t]{2}{*}{ RT delivery } & Non-IGRT & 94 & $(85)$ & 96 & $(87)$ & 0.05 \\
\hline & IGRT & 16 & (15) & 14 & (13) & \\
\hline \multirow[t]{2}{*}{ Institution } & Low volume & 40 & (36) & 45 & $(41)$ & 0.09 \\
\hline & High volume & 70 & $(64)$ & 65 & (59) & \\
\hline \multirow[t]{2}{*}{ Use of PET } & Without & 12 & $(11)$ & 12 & $(11)$ & 0.00 \\
\hline & With & 98 & (89) & 98 & (89) & \\
\hline \multirow[t]{2}{*}{ Location } & Cervical & 1 & (1) & 1 & $(1)$ & 0.00 \\
\hline & Noncervical & 109 & (99) & 109 & (99) & \\
\hline
\end{tabular}

sd standard deviation, RT radiotherapy, IGRT image-guided RT, PET positron emission tomography, CCRT concurrent chemoradiotherapy ${ }^{\mathrm{a}}$ Rounded

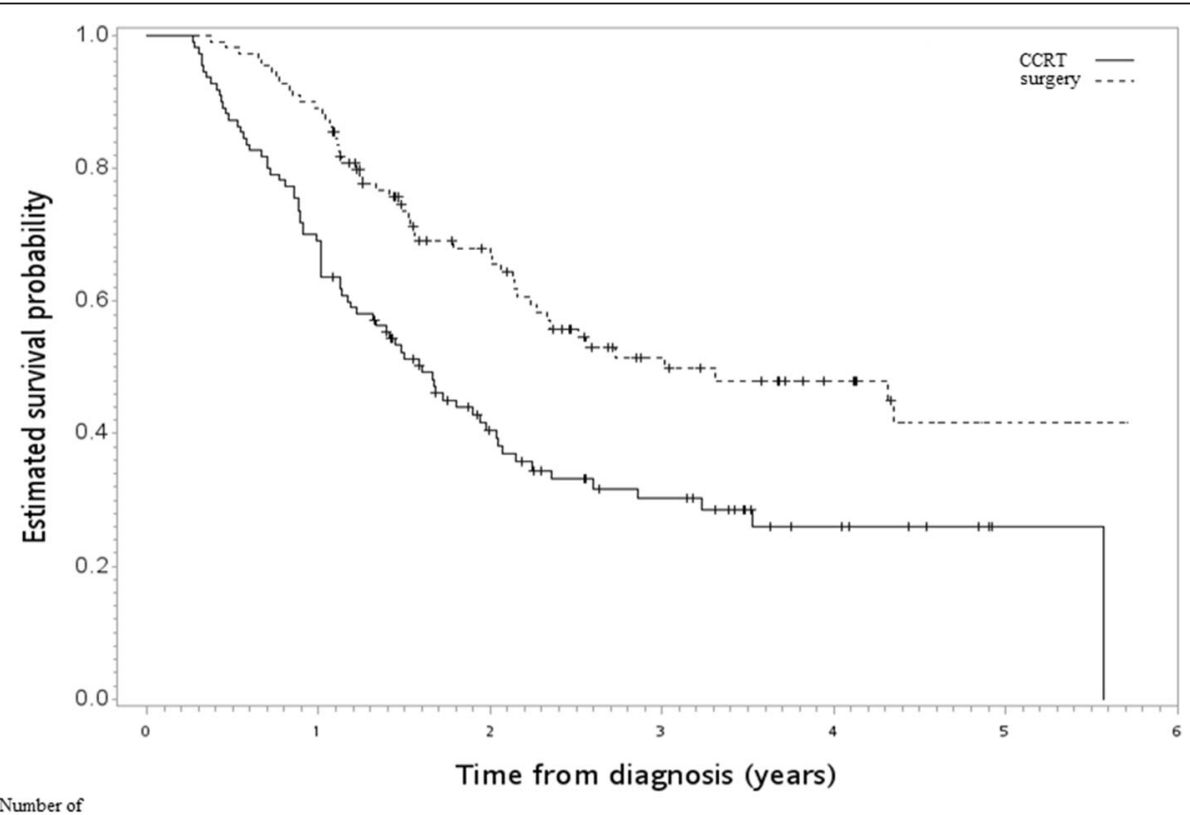

Number of

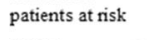

$\begin{array}{ll}\text { CCRT } & 110 \\ \text { surgery } & 110\end{array}$

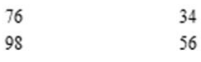

$\begin{array}{ll}34 & 20 \\ 56 & 30\end{array}$

$\begin{array}{rrr}8 & 1 & 0 \\ 19 & 7 & 0\end{array}$

Fig. 3 Kaplan-Meier survival curve (in years) for a PS-matched subgroup diagnosed from 2011 to 2015 [SA-1] 
Table 3 Characteristics of the matched study population in the SA-2

\begin{tabular}{|c|c|c|c|c|c|c|}
\hline & & \multicolumn{2}{|l|}{ Surgery [MIE] } & \multicolumn{2}{|l|}{ CCRT } & \multirow{2}{*}{$\begin{array}{l}\text { Standardized } \\
\text { difference }^{a}\end{array}$} \\
\hline & & Number or mean $(s d)^{a}$ & $(\%)^{a}$ & Number or mean $(\mathrm{sd})^{\mathrm{a}}$ & $(\%)^{a}$ & \\
\hline Age & & $56.38(8.21)$ & & $55.35(10.58)$ & & 0.11 \\
\hline \multirow[t]{2}{*}{ Gender } & Female & 4 & (5) & 4 & (5) & 0.00 \\
\hline & Male & 78 & $(95)$ & 78 & $(95)$ & \\
\hline \multirow[t]{2}{*}{ Residency } & Non-north & 57 & $(70)$ & 52 & $(63)$ & 0.13 \\
\hline & North & 25 & $(30)$ & 30 & $(37)$ & \\
\hline \multirow[t]{2}{*}{ Tumor size } & $\leq 5 \mathrm{~cm}$ & 31 & (38) & 31 & (38) & 0.00 \\
\hline & $>5 \mathrm{~cm}$ & 51 & $(62)$ & 51 & $(62)$ & \\
\hline \multirow[t]{2}{*}{ Differentiation } & Poorly/undifferentiated & 31 & $(38)$ & 30 & $(37)$ & 0.03 \\
\hline & Well/moderately & 51 & $(62)$ & 52 & $(63)$ & \\
\hline \multirow[t]{2}{*}{ RT delivery } & Non-IGRT & 70 & $(85)$ & 68 & (83) & 0.07 \\
\hline & IGRT & 12 & $(15)$ & 14 & $(17)$ & \\
\hline \multirow[t]{2}{*}{ Institution } & Low volume & 23 & $(28)$ & 25 & (30) & 0.05 \\
\hline & High volume & 59 & $(72)$ & 57 & $(70)$ & \\
\hline \multirow[t]{2}{*}{ Use of PET } & Without & 7 & (9) & 11 & (13) & 0.16 \\
\hline & With & 75 & (91) & 71 & $(87)$ & \\
\hline
\end{tabular}

The nonoverlapped covariable (location) was not included in this matching

sd standard deviation, $R T$ radiotherapy, IGRT image-guided RT, PET positron emission tomography, MIE minimally invasive esophagectomy, CCRT

concurrent chemoradiotherapy

${ }^{\mathrm{a}}$ Rounded

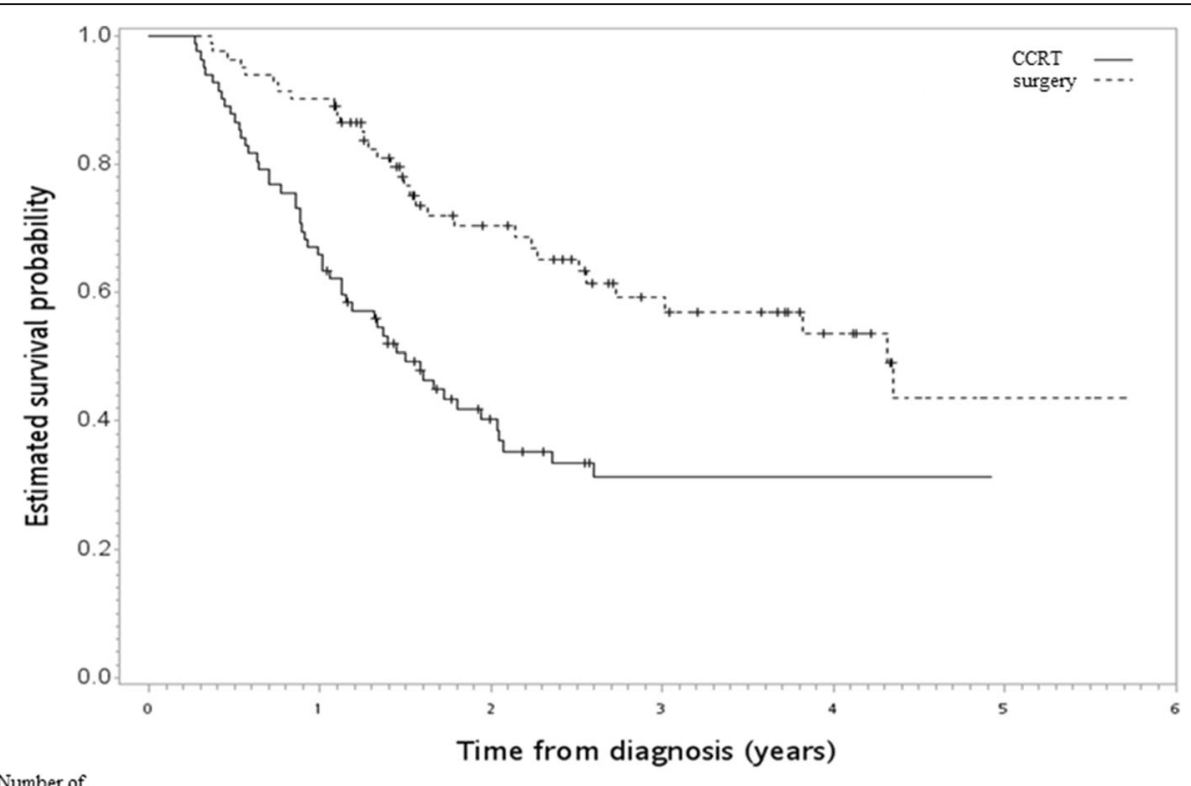

Number of

patients at risk

$\begin{array}{llllllll}\text { CCRT } & \$ 2 & 54 & 24 & 12 & 8 & 0 & 0 \\ \text { surgery } & 82 & 74 & 42 & 25 & 15 & 4 & 0\end{array}$

Fig. 4 Kaplan-Meier survival curve (in years) for a PS-matched subgroup diagnosed from 2011 to 2015, but with the surgery group limited to those who received MIE [SA-2] 


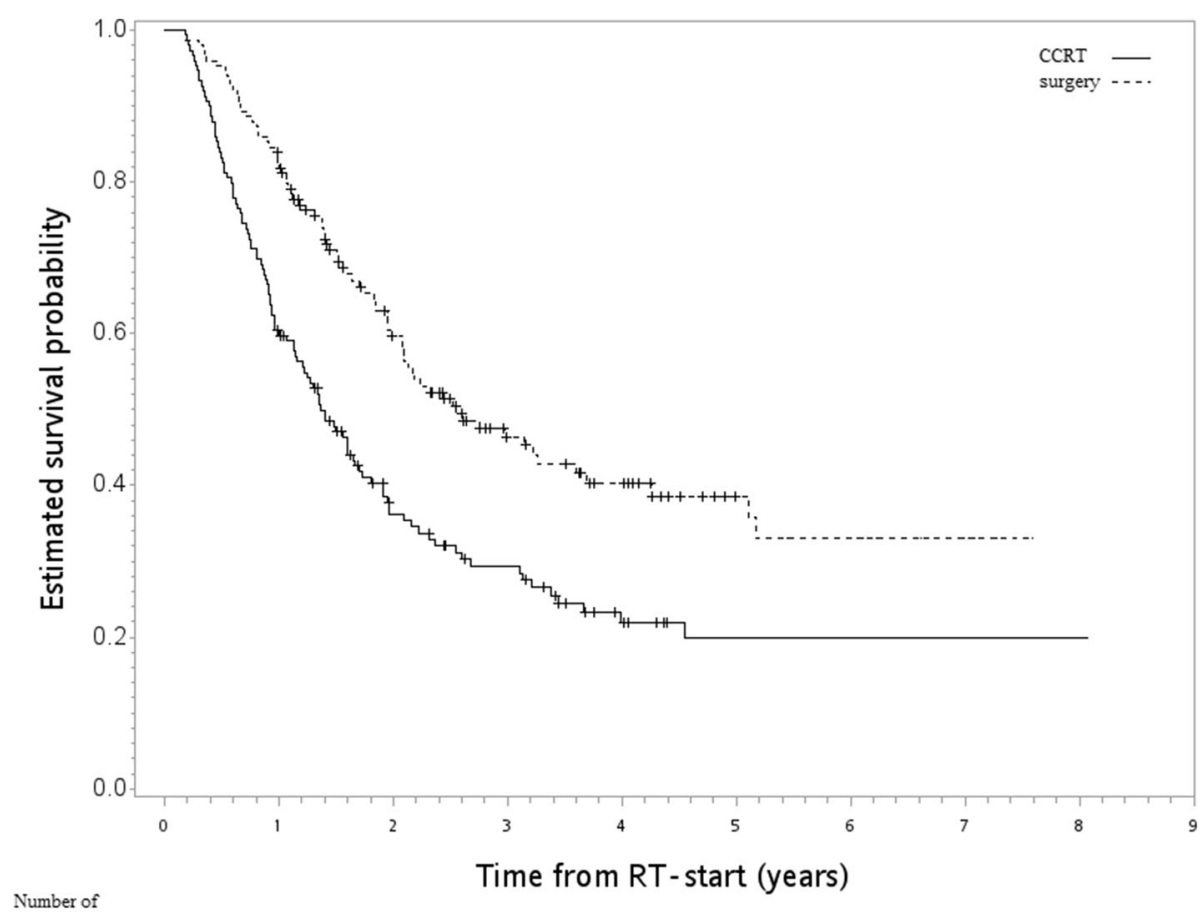

Fig. 5 Kaplan-Meier survival curve (in years) for the final study population when the index date was changed to the start of radiotherapy [SA-3]

Statistically, our results were not consistent with available RCTs [6, 7]; however, survival was actually numerically better for the surgical arm when compared to that for the nonsurgical arms in both RCTs, although not statistically significant. The 2-year OS was 39.9 vs $35.4 \%$ in the German trial [7] and 37.1 vs $36.5 \%$ [per protocol] in the French trial [6]. In our study, it was 63 vs $39 \%$. Therefore, the outcome of CCRT in our study was similar to both RCTs, but the outcome of neoadjuvant CCRT followed by esophagectomy was higher in our study [63\%] (although close to the 67\% OS reported in the modern CROSS study [5]).

We feel that our findings are compatible with the prevalent concept that esophagectomy is an integral part of treatment for locally advanced esophageal cancer [1], possibly through improved disease control (demonstrated by the improved FFLRR and ECSS), as better local/regional control in the surgical arm had also been reported in the above RCTs $[6,7]$. However, given the abovementioned RCTs and that our result was sensitive to potential unmeasured confounders [E-factor 1.74], we feel that our results should not be interpreted as conclusive and that further modern RCTs are needed. When we searched in "https://clinicaltrials.gov/" using ["esophagectomy" AND "concurrent chemoradiotherapy"] on May 21, 2018, we found two relevant ongoing trials [NCT02972372, NCT01740375]; however, these trials were relevant but not identical. NCT02972372 investigated definitive CCRT vs surgery without neoadjuvant CCRT, whereas NCT01740375 investigated upfront surgery or observation for those patients who achieved complete clinical response. Therefore, the results of our study could be valuable for contemporary patient decision-making until other modern high-level evidence becomes available.

There were some limitations in our study. First, we shared the inherent limitation of observation study. Although we tried our best to follow the STROBE guidelines for observational study [10] and examined extensive supplementary analyses, potential unmeasured confounder(s), such as presenting body weight loss [6] or operability, might still threaten our results. Second, the generalizability of our finding to regions where $\mathrm{SqCC}$ was not the predominant histology might be questionable.

\section{Conclusions}

We found that for locally advanced esophageal SqCC, neoadjuvant CCRT followed by esophagectomy was associated with improved OS when compared to CCRT without surgery. However, given the nonrandomized nature of the study and sensitivity to potential unmeasured confounder(s), our results should be interpreted cautiously. 


\section{Abbreviations}

CCRT: Concurrent chemoradiotherapy; ECSS: Esophageal cancer-specific survival; FFLRR: Freedom from local regional recurrence; IGRT: Image-guided radiotherapy; MIE: Minimally invasive esophagectomy; OS: Overall survival; PET: Positron emission tomography; PS: Propensity score; RCT: Randomized controlled trial; RT: Radiotherapy; SA: Supplementary analysis; SqCC: Squamous cell carcinoma; TCR: Taiwan Cancer Registry

\section{Acknowledgements}

The authors are thankful for the conceptual input from Dr. Tsang-Wu Liu and all of the members in the Health Promotion Administration, National Health Research Institute Esophageal Cancer Committee for their conceptual contributions. The authors thank "American Journal Experts" for editorial assistance. The corresponding author would like to thank Dr. Ya-Chen Tina Shih for her mentoring in Health Services Research.

\section{Funding}

This work was supported by the Health Promotion Administration, Ministry of Health and Welfare, Taiwan (R.O.C.) [source of this funding is the health and welfare surcharge of tobacco products] and China Medical University Hospital [CRS-106-040 to Chien C.R.]

\section{Availability of data and materials}

The data that support the findings of this study are available from the Taiwan Cancer Registry; however, restrictions apply to the availability of these data, which were used under license for the current study, and so are not publicly available. Data are, however, available from the authors upon reasonable request and with permission of the Taiwan Cancer Registry.

\section{Authors' contributions}

CYC participated in the conceptualization and design of the study, interpreted data, and drafted the manuscript. CCL participated in the conceptualization and design of study, analyzed data, and drafted the manuscript. CRC participated in the conceptualization and design of study, collected the related studies, analyzed and interpreted data, and drafted the manuscript. All authors have read and approved the final manuscript.

\section{Ethics approval and consent to participate}

This study was approved by Research Ethics Committee, National Health Research Institutes [EC1041006-E].

\section{Consent for publication}

Not applicable.

\section{Competing interests}

The authors declare that they have no competing interests.

\section{Publisher's Note}

Springer Nature remains neutral with regard to jurisdictional claims in published maps and institutional affiliations.

\section{Author details}

'Division of Thoracic Surgery, Department of Surgery, Chung Shan Medical University, Chung Shan Medical University Hospital, Taichung, Taiwan. ${ }^{2}$ Department of Radiation Oncology, China Medical University Hospital, Taichung, Taiwan. ${ }^{3}$ Department of Radiation Oncology, China Medical University Hsinchu Hospital, Hsinchu, Taiwan. ${ }^{4}$ School of Medicine, College of Medicine, China Medical University, No.91 Hsueh-Shih Road, North District, Taichung 40402, Taiwan.

\section{Received: 23 January 2018 Accepted: 5 July 2018}

Published online: 14 July 2018

\section{References}

1. Rustgi AK, El-Serag HB. Esophageal carcinoma. N Engl J Med. 2014;371: 2499-509.

2. National Comprehensive Cancer Network. National Comprehensive Cance Network Guidelines for esophageal and esophagogastric junction cancers, version 3.2017 [free registration required]. https://www.nccn.org/ professionals/physician_gls/pdf/esophageal.pdf. Accessed 30 Sep 2017.
3. Chun SG, Skinner HD, Minsky BD. Radiation therapy for locally advanced esophageal cancer. Surg Oncol Clin N Am. 2017:26:257-76.

4. Sjoquist KM, Burmeister BH, Smithers BM, Zalcberg JR, Simes RJ, Barbour A, et al. Survival after neoadjuvant chemotherapy or chemoradiotherapy for resectable oesophageal carcinoma: an updated meta-analysis. Lancet Oncol. 2011;12:681-92.

5. Shapiro J, van Lanschot JJB, Hulshof MCCM, van Hagen P, van Berge Henegouwen MI, Wijnhoven BPL, et al. Neoadjuvant chemoradiotherapy plus surgery versus surgery alone for oesophageal or junctional cancer (CROSS): long-term results of a randomised controlled trial. Lancet Oncol. 2015;16:1090-8.

6. Bedenne L, Michel P, Bouché O, Milan C, Mariette C, Conroy T, et al. Chemoradiation followed by surgery compared with chemoradiation alone in squamous cancer of the esophagus: FFCD 9102. J Clin Oncol. 2007;25:1160-8.

7. Stahl M, Stuschke M, Lehmann N, Meyer HJ, Walz MK, Seeber S, et al. Chemoradiation with and without surgery in patients with locally advanced squamous cell carcinoma of the esophagus. J Clin Oncol. 2005:23:2310-7.

8. Chiu PW, Chan AC, Leung SF, Leong HT, Kwong KH, Li MK, et al. Multicenter prospective randomized trial comparing standard esophagectomy with chemoradiotherapy for treatment of squamous esophageal cancer: early results from the Chinese University Research Group for Esophageal Cancer (CURE). J Gastrointest Surg. 2005:9:794-802.

9. Chiang CJ, You SL, Chen CJ, Yang YW, Lo WC, Lai MS. Quality assessment and improvement of nationwide cancer registration system in Taiwan: a review. Jpn J Clin Oncol. 2015;45:291-6.

10. von Elm E, Altman DG, Egger M, Pocock SJ, Gøtzsche PC, Vandenbroucke $J P$, et al. The Strengthening the Reporting of Observational Studies in Epidemiology (STROBE) statement: guidelines for reporting observational studies. Int J Surg. 2014;12:1495-9.

11. Chen CY, Li CC, Chien CR. Does higher radiation dose lead to better outcome for non-operated localized esophageal squamous cell carcinoma patients who received concurrent chemoradiotherapy? A population based propensity-score matched analysis. Radiother Oncol. 2016;120:136-9.

12. Li CC, Chen CY, Chien CR. Comparative effectiveness of image-guided radiotherapy for non-operated localized esophageal squamous cell carcinoma patients receiving concurrent chemoradiotherapy: a populationbased propensity score matched analysis. Oncotarget. 2016;7:71548-55.

13. Fang HY, Chen CY, Wang YC, Wang PH, Shieh SH, Chien CR. Consistently lower narcotics consumption after video-assisted thoracoscopic surgery for early stage non-small cell lung cancer when compared to open surgery: a one-year follow-up study. Eur J Cardiothorac Surg. 2013;43:783-6.

14. Austin PC. The use of propensity score methods with survival or time-toevent outcomes: reporting measures of effect similar to those used in randomized experiments. Stat Med. 2014;33:1242-58.

15. Jagsi R, Bekelman JE, Chen A, Chen RC, Hoffman K, Shih YC, et al. Considerations for observational research using large data sets in radiation oncology. Int J Radiat Oncol Biol Phys. 2014;90:11-24.

16. Gockel I, Ahlbrand CJ, Arras M, Schreiber EM, Lang H. Quality management and key performance indicators in oncologic esophageal surgery. Dig Dis Sci. 2015;60:3536-44.

17. Garrido MM, Kelley AS, Paris J, Roza K, Meier DE, Morrison RS, et al. Methods for constructing and assessing propensity scores. Health Serv Res. 2014;49:1701-20.

18. Ali MS, Groenwold RH, Belitser SV, Pestman WR, Hoes AW, Roes KC, et al. Reporting of covariate selection and balance assessment in propensity score analysis is suboptimal: a systematic review. J Clin Epidemiol. 2015;68:112-21.

19. VanderWeele TJ, Ding P. Sensitivity analysis in observational research: introducing the E-value. Ann Intern Med. 2017:167:268-74.

20. Yibulayin W, Abulizi S, Lv H, Sun W. Minimally invasive oesophagectomy versus open esophagectomy for resectable esophageal cancer: a metaanalysis. World J Surg Oncol. 2016;14:304.

21. Straatman J, van der Wielen N, Cuesta MA, Daams F, Roig Garcia J, Bonavina $L$, et al. Minimally invasive versus open esophageal resection: three-year follow-up of the previously reported randomized controlled trial: the TIME trial. Ann Surg. 2017;266:232-6. 